expiratory volume in 1 second $\left(\mathrm{FEV}_{1}\right)$ as well as exocrine pancreatic marker faecal elastase-1 (FE-1) and random blood glucose within the Ivacaftor group.

Results The change in pancrealipase use was significantly decreased in the Ivacaftor group in comparison to controls $(1202 \pm 587 \mathrm{IU} / \mathrm{kg}, \mathrm{p}=0.039)$. In addition, Ivacaftor users had a significant increase in gross BMI $\left(0.98 \pm 0.51 \mathrm{~kg} / \mathrm{m}^{2}\right.$, $\mathrm{p}=0.010)$ and a non-significant increase in $\mathrm{FEV}_{1} \%$ predicted $(10.9 \pm 5.06 \%, p=0.123)$ in comparison to controls. On subgroup analysis, there was significant improvement in FE-1 after one year of Ivacaftor use $(107 \pm 80.8 \mu \mathrm{g} / \mathrm{g}, \mathrm{p}=0.013$, $\mathrm{N}=7$ ). As well, there was mild decrease seen in random blood glucose, however this result was not significant $(-0.43 \pm 0.87$ $\mathrm{mmol} / \mathrm{L}, \mathrm{p}=0.153, \mathrm{~N}=10$ ).

Conclusions Ivacaftor improves paediatric CF patients' BMI, blood glucose, and FE-1 values while reducing their reliance on pancrealipase. This data supports previous research showing increasing lung function in Ivacaftor users. Subgroup analysis of those with serial FE-1 results revealed that $43 \%$ of those taking Ivacaftor reached pancreatic exocrine sufficiency $(\mathrm{FE}-1>200 \mu \mathrm{g} / \mathrm{g})$. This implies that further study of Ivacaftor's pancreatic implications is warranted. Similarly, prospective studies of new and emerging drugs of this class in a similar manner could yield positive results for patients.

\section{GP285 THE EFFECT OF MODULATOR THERAPY ON LIVER FUNCTION IN PATIENTS WITH CYSTIC FIBROSIS- RELATED LIVER DISEASE (CFLD)}

\footnotetext{
'Sharon Dempsey*, 'John Travers, 'Gerardine Leer, 'Geraldine Connell, 'Shona Quinn, 1,2Peter Greally, 1,2Basil Elnazir. 'Children's Hospital Ireland, Tallaght, Dublin, Ireland; ${ }^{2}$ Trinity College, Dublin, Ireland
}

10.1136/archdischild-2019-epa.344

Introduction Cystic Fibrosis transmembrane conductance regulator (CFTR) modulator therapies are utilised to correct the malfunctioning protein made by the CFTR gene thus producing significant improvement in lung function. However, worsening liver function is described as a potentially serious side effect by modulator suppliers.

ORKAMBI and IVACAFTOR are licensed for the treatment of cystic fibrosis (CF) in patients greater than 6 years who are homozygous for the F508del-CFTR mutation. We set out to identify changes to liver function and pathology after initiation of modulator therapy in a retrospective study of all CFLD patients.

Methodology We reviewed the liver function tests and liver ultrasound results on all patients with CFLD. We recorded anonymised test results for ALT, AST, Alk Phos, GGT and bilirubin from birth. We reviewed patient files and recorded medication histories. We mapped changes in medication against chronological liver function tests and identified trends of improving or worsening results.

Results Ninety-eight children with Cystic Fibrosis attend Tallaght. Thirteen have CFLD (13\%) of which 54\% are female. The predominant phenotype is $\Delta$ Phe508del/APhe508del (9/ $13,69 \%)$. $\Delta$ Phe508del/G551D was isolated in two children (15\%), $\Delta$ Phe508del/V520F in one child $(8 \%)$ and $\Delta$ Phe508del/Arg560 Thr/Lys heterozygous in one child $(8 \%)$. Seven children $(7 / 13,54 \%)$ take ORKAMBI and two $(2 / 13$, $15 \%)$ take IVACAFTOR. Four children with CFLD are not currently taking modulator therapy due to their specific phenotype, age or social circumstances. We identified an improvement in liver function tests in $89 \%(8 / 9)$ since initiation of therapy - $86 \%$ taking ORKAMBI and 100\% taking IVACAFTOR. One patient on ORKAMBI $(1 / 7,14 \%)$ had no improvement in liver function tests, with persistently raised GGT and AST, which began prior to modulator therapy initiation. $100 \%$ of the ORKAMBI cohort showed no deterioration in liver pathology over 18 months. 50\% of the IVACAFTOR cohort demonstrated no deterioration in liver pathology over 6 years.

Conclusions and discussion We identified an improvement in liver function tests in $89 \%$ of children with CFLD since the commencement of modulator therapy. Limitations include the small sample size and variance in time taking modulator therapy (from 6 months to six years). Further study would be helpful to reconcile the variance between supplier side effect warnings and these results.

\section{GP286 CAN FOLLOW UP RENAL ULTRASOUND REPLACE DMSA FOR DETECTION OF PARENCHYMAL CHANGES IF FIRST ULTRASOUND POST URINARY TRACT INFECTION IN CHILDREN IS NORMAL? AN AUDIT OF NATIONAL UTI GUIDELINES OF IRELAND}

${ }^{1,2}$ Muhammad Rizwan*, ${ }^{3}$ Louise Gibson, ${ }^{4}$ Micheal Moore. ${ }^{1}$ Paediatrics Hull Royal Infirmary, Hull, UK; ${ }^{2}$ Ex Registrar Paediatrics Cork university Hospital, Cork, Ireland; ${ }^{3}$ Paediatrics Cork university Hospital, Cork, Ireland; ${ }^{4}$ Radiology Cork university Hospital, Cork, Ireland

\subsection{6/archdischild-2019-epa.345}

Background Urinary tract infections (UTI) in Children can cause serious morbidity as well as Scarring of kidneys leading to renal impairment. To manage UTI in children and avoid the bad outcome, there are different guidelines for close follow up and management of these children. There were new Irish guidelines set up in 2017 regarding imaging of these children which suggested Renal ultrasound (US) at admission of clinically well children with UTI followed by the Renal US after 6 months instead of DMSA if the initial US is normal.

Method We gathered the data of all sick children under 16 years of age from January 2014-Dec 2016, who were diagnosed with Culture proven UTI and had Renal US at admission and DMSA or Renal US at follow up. We identified 70 (39 male- 55.7\%, 31 females-44.3\%) cases who had all the data available on Microbiology, Radiology and medical notes, and excluded all those who were either out of catchment area, who lost follow up appointments, or whose report was not traced properly. In 70 children admitted there $32(45.7 \%)$ who had repeat US along with DMSA. The reports were verified by Expert Pediatric Radiologist.

Results We found 62 (88.5\%) normal initial US (Normal parenchyma), who had normal DMSA and repeat US (24 cases). There were $8(11.5 \%)$ children who had abnormal initial US (parenchymal changes), which were confirmed by DMSA and repeat US (8 cases).

Conclusion Renal US could be a potential alternative of DMSA in children with UTI if the initial US has been reported normal if the scans are reported by Pediatric radiologist/Special interest in pediatric radiology. The renal US is cost and time effective, and in this way, we can avoid extra costs, day admissions and family anxiety related to the procedure. 